\title{
Medical Analysis and Diagnosis by Neural Networks
}

\author{
Rüdiger W. Brause \\ J.W. Goethe-University, Computer Science Dept., Frankfurt a. M., Germany. \\ Brausedcs.uni-frankfurt.de
}

\begin{abstract}
.
In its first part, this contribution reviews shortly the application of neural network methods to medical problems and characterizes its advantages and problems in the context of the medical background. Successful application examples show that human diagnostic capabilities are significantly worse than the neural diagnostic systems. Then, paradigm of neural networks is shortly introduced and the main problems of medical data base and the basic approaches for training and testing a network by medical data are described. Additionally, the problem of interfacing the network and its result is given and the neuro-fuzzy approach is presented. Finally, as case study of neural rule based diagnosis septic shock diagnosis is described, on one hand by a growing neural network and on the other hand by a rule based system.
\end{abstract}

\section{Keywords}

Statistical Classification, Adaptive Prediction, Neural Networks, Neurofuzzy Medical Systems

\section{Introduction}

Almost all the physicians are confronted during their formation by the task of learning to diagnose. Here, they have to solve the problem of deducing certain diseases or formulating a treatment based on more or less specified observations and knowledge. Certainly, there is the standard knowledge of seminars, courses and books, but on one hand medical knowledge outdates quickly and on the other hand this does not replace own experience. For this task, certain basic difficulties have to be taken into account:

- The basis for a valid diagnosis, a sufficient number of experienced cases, is reached only in the middle of a physician's career and is therefore not yet present at the end of the academic formation.

- This is especially true for rare or new diseases where also experienced physicians are in the same situation as newcomers.

- Principally, humans do not resemble statistic computers but pattern recognition systems. Humans can recognize patterns or objects very easily but fail when probabilities have to be assigned to observations. 
These principal difficulties are not widely known by physicians. Also studies who revealed that about $50 \%$ of the diagnoses are wrong do not impede the selfconscience of some physicians. It is not by chance that the disease AIDS which manifests by a myriad of infections and cancer states was not discovered directly by treating physicians but by statistical people observing the improbable density of rare cancer cases at the U.S. west coast.

An important solution for the described problem lies in the systematic application of statistical instruments. The good availability of computers ameliorate the possibilities of statistically inexperienced physicians to apply the benefits of such a kind of diagnosis:

- Also physicians in the learning phase with less experience can obtain a reliable diagnosis using the collected data of experienced colleagues.

- Even in the case of rare diseases, e.g. septic shock, it is possible to get a good diagnosis if they use the experience of world-wide networked colleagues.

- New, unknown diseases can be systematically documented even if this induces complex computations which are not known to the treating physician.

- Also in the treatment of standard diseases a critical statistical discussion for the use of operation methods or medical therapies may introduce doubts in the physicians own, preferred methods as it is propagated by the ideas of evidence based medicine EBM[16].

A classical, early study [8] in the year 1971 showed these basic facts in the medical area. At the university clinic of Leeds (UK) 472 patients with acute abdominal pain where examined and diagnosed. With simple, probability-based methods (Bayes classification) the diagnostic decision probabilities were computed based on a data base of 600 patients. Additionally, a second set of probabilities were computed by using a synthetic data base of patients build on the interviews of experts and questionnaire sheets about 'typical' symptoms.

Then, the 472 cases were diagnosed by an expert round of 3 experienced and 3 young physicians. The results of this experiment was as follows:

- Best human diagnosis (most experienced physician): $79.7 \%$

- Computer with expert data base: $82.2 \%$

- Computer with 600 patient data: $91.1 \%$

The conclusion is clear: humans can not ad hoc analyze complex data without errors. Can neural networks help in this situation?

\section{The Prognostic Capabilities of Neural Networks}

Let us shortly review the prognostic capabilities of adaptive systems like those of neural networks. There is a long list of successful applications of neural networks in medicine, e.g. [13],[27]. Examples are given below: 
- Myocardial infarction [1]

From 356 patients of a heart intensive care unit 120 suffered from acute myocardial infarction. Based on these data, Baxt (1990) trained a network and obtained a sensitivity of $92 \%$ and a specificity of $96 \%$ for heart attack prediction.

- back pain [3]

145 responses of a questionnaire represented the input, 4 possible diagnosis results were the output (simple lower back pain SLBP, root pain RP, spinal pain $\mathrm{SP}$, abnormal illness behavior AIB). After training with 50 example cases the following correct percentage for 50 test cases were observed (Table 1):

Table 1 Diagnostic correctness of back pain.

\begin{tabular}{l|cccc|c} 
Method & SLBP \% & RP \% & SP \% & AIB \% & average \% \\
\hline Network & 63 & 90 & 87 & 95 & 83 \\
neuro-surgeon & 96 & 92 & 60 & 80 & 82 \\
orthoped. surg. & 88 & 88 & 80 & 80 & 84 \\
common phys. & 76 & 92 & 64 & 92 & 81
\end{tabular}

For this application, the network has (in the average) roughly the same success as the human, experienced experts. Nevertheless, for the non-critical case of simple lower back pain the network was worse than the physicians; for the important case of spinal symptoms where a quick intervention is necessary the network was better than the experts.

- Survival probability after severe injury [21]

For 3 input variables (Revised Trauma Score RTS, Injury Severity Score ISS, age) and 2 output variables (life, death) a network was trained with 4800 examples. Compared to the traditional score method TRISS and a variant ASCOT which separate special risk groups before scoring, resulted in the following diagnostic scores for juvenile patients (Table 2):

Table 2 Diagnostic success for severe injury of juvenile patients

\begin{tabular}{l|ccc} 
Diagnose & TRISS & ASCOT & NNet \\
\hline sensitivity \% & 83,3 & 80,6 & 90,3 \\
specificity \% & 97,2 & 97,5 & 97,5
\end{tabular}

The significant higher sensitivity of the neural network can be deduced to the superiority of the adaptive approach of the neural net compared to the fixed linear weighting of the scores (as e.g. APACHE). A linear weighting corresponds to only one layer of linear neurons (e.g. the output layer); the categorical score input corresponds to fixed nonlinear neurons (e.g. the hidden units).

Beside the high number of successful medical applications (MedLine [18] listed about 1700 papers for the keywords "artificial neural network" in spring 2001) 
there are many reviews for the use of artificial neural networks in medicine, see e.g. [9],[24],[26]. In this contribution, only the basic principles of neural networks will be presented in the next section in order to set the base for applications like the one in section 4 .

\section{Basic Principles of Neural Networks}

Let us start by modelling the artificial neurons. Like in nature neural networks consist of many small units, the formal neurons. They are interconnected and work together. Each neuron has several inputs and one output only. In Fig. 1 a biological neuron and an artificial neuron are shown.
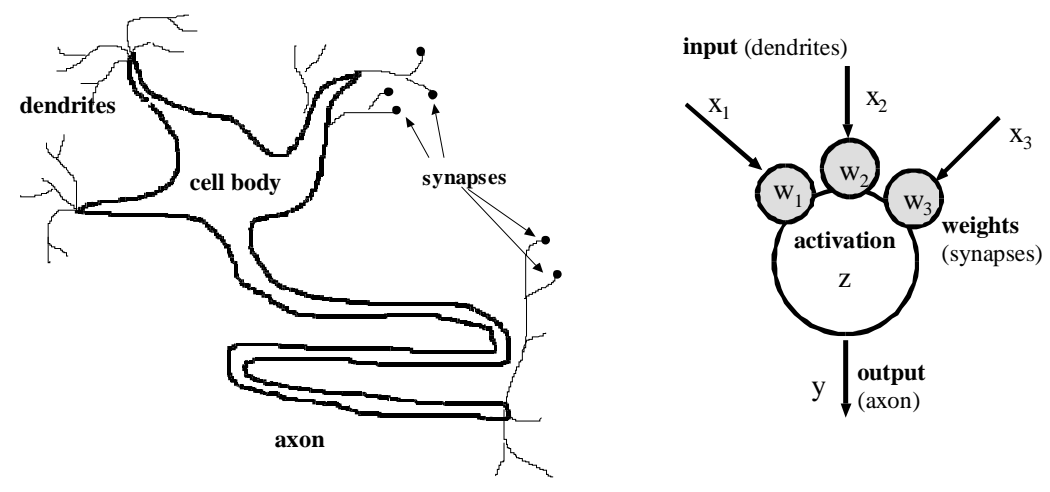

Fig. 1 A biological neuron and an artificial one.

Our formal neuron has inputs $\mathrm{x}_{\mathrm{i}}$, each one weighted by a weight factor $\mathrm{w}_{\mathrm{i}}$. We model all of the neural inputs from the same neighbour neuron by just one weighted input. Typically, the activation $z$ is modelled by a weighted sum of the $n$ inputs

$$
\mathrm{z}=\sum_{\mathrm{i}=1}^{\mathrm{n}} \mathrm{w}_{\mathrm{i}} \mathrm{x}_{\mathrm{i}}
$$

The output activity $y$ is a function $S$ of the activation, generally a nonlinear one.

Nonlinear predictions are provided by nonlinear neurons, i.e. neurons with a nonlinear function $\mathrm{S}_{\mathrm{i}}(\mathrm{z})$ for the $\mathrm{i}$-th neuron, e.g. a radial basis function $(\mathrm{RBF})$

$$
\mathrm{S}_{\mathrm{i}}(\mathrm{z})=\mathrm{e}^{-\mathrm{z}^{2}} \text { with } \mathrm{z}^{2}=\frac{\left(\mathbf{c}_{i}-\mathbf{x}\right)^{2}}{2 \sigma_{i}{ }^{2}}
$$

This bell-shaped function provides a local sensitivity of each neuron $i$ for an area of width $\sigma_{i}$ centred at point $\mathbf{c}_{\mathrm{i}}$.

If we arrange several neurons in parallel and then in different layers, we get a mapping from input to output ("feedforward network"). Given a certain task, what 
kind of network should we choose? To resolve this question, we should know: what is in general the power of a network? For a two layer network (Fig. 2) containing at least one nonlinear layer we know that we can approximate any function as close as desired. For a more precise notation of this property, see e.g. [15].

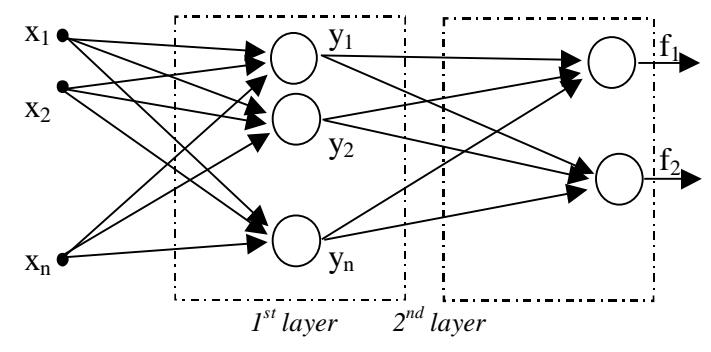

Fig. 2 A two-layer network

For our purpose, we have to decide whether we want to solve a classification or prediction task, based on a number of known cases, or if we want to make a kind of data mining approach, discovering new proportions of the data. In the first case we should take a multi-layer decision network with a learning algorithm based on the classification probability, not on a distance measure like the mean square error of approximation. The classification might be done either by a multi-layerperceptron MLP or a radial basis function network RBF, see [12]. In either case, the network is trained to do a certain classification job by presenting the patient data and the correct classification to the network. It is the task of the network to predict the class of an unknown patient from the presented data, giving rise to appropriate treatments.

\section{Preprocessing the data}

Very important for medical data analysis, especially for retrospective evaluations, is the preprocessing of the data, see [23]. The problems are listed below.

- The data set in single studies is often too small to produce reliable results.

- Often, medical data material is very inhomogeneous, coming from multivariate time series with irregularly measured time stamps.

- Typing errors are detected by checking bounds of the variables.

- A lot of variables shows a high number of missing values caused by faults or simply by seldom measurements.

- Feature variables should be selected to avoid the so called "curse of dimensionality"

For our task we heavily rely on the size of the data and their diagnostic quality. If the data contains too much inaccurate or missing entries we have no chance of building up a reliable system even if it is principally possible. 


\section{Training and Testing}

In general, the networks have to be trained in order to get the parameters set for a proper function. We distinguish between two modes: the supervised training where we add to each training sample input (patient data) also the desired network output information (e.g. the correct classification), and the unsupervised training which is used to extract statistical information from the samples. The latter is often used for signal preprocessing, e.g. PCA and ICA, see [12].

How do we get the parameters of the chosen network, e.g. $\sigma_{\mathrm{i}}$ and $\mathbf{c}_{\mathrm{k}}$ of eq.(2)? 1 Changing the parameters at fixed network: The parameters are updated such that an objective function $\mathrm{R}(\mathrm{w})$ is optimised. 2 Growing networks with fixed parameters: Starting with one neuron, for each data sample which causes a high error in the prediction a new neuron is added to the network. All parameters are set such that the error is decreased.

It is well known that the performance of learning systems on the training data often does not reflect the performance on unknown data. This is due to the fact that the system often adapts well on training to the particularities of the training data. Therefore, the training data should be randomly chosen from all available data. It should represent the typical data properties, e.g. the probability distribution. If you have initially a bias in the training data you will encounter performance problems for the test data later.

In order to test the real generalization abilities of a network to unknown data, it must be tested by classified, but yet unknown data, the test data that should not contain samples coming from patients of the training data. We have to face the fact that patient data is very individual and it is difficult to generalize from one patient to another. Ignoring this fact would pretend better results than a real system could practically achieve.

\section{Interfacing the Results}

One of the most important questions for diagnosis is the design of the user interface. Why?

Neural networks are seldom designed to explain what they have learned. The approach of using the experience of the physician and explaining the diagnosis by proper medical terms is crucial for the question whether a diagnostic system is used or ignored. In general, all diagnostic systems, even the most sophisticated ones, are worthless if they are not used. So, the importance of acquiring the necessary knowledge and presenting the results in a human understandable, easy way can not be overestimated.

Now, with the appearance of fuzzy systems which use vague, human-like categories [20] the situation for knowledge-based diagnosis has changed. Based on the well-known mechanisms of learning in RBF networks, a neuro-fuzzy interface can be used for the input and output of neural systems. The intuitive and instructive interface is useful in medical applications, using the notation and habits of physicians and other medically trained people. In Fig. 3 this concept is visualized. 


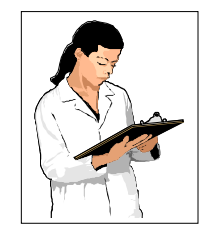

physician

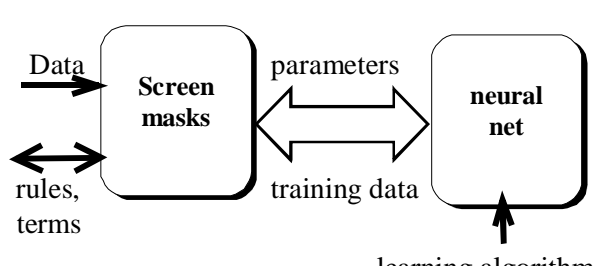

learning algorithm

Fig. 3 Interactive transfer of vague knowledge

Here, the user interface must use the typical human properties and formulate the diagnosis by the vague, inexact language of physicians. The following notational habits of physicians for variables and possible outcomes have to be reflected by the user interface:

- Exact notation, e.g. blood sugar $=120 \mathrm{mg} / \mathrm{dl}$.

- Semi-quantitative notation, e.g. 120 to $130 \mathrm{mg} / \mathrm{dl}$ or ++, +, 0, -, -- .

- Qualitative, categorical notation, e.g. test result $=$ red.

To support these notations, we might use the idea of fuzzy terms, called "vague terms", described by membership functions. As example, in Fig. 4 the assignment of the vague linguistic terms of the medical variable SGOT to the vague variable $\mathrm{x}$ (concentration IE/l) is shown. This results in a vague set of membership functions showed in Fig. 4. For each function, the set $\{x \mid \mu(x)=1\}$ is called the core, whereas the whole set $\{\mathrm{x} \mid \mu(\mathrm{x})>0\}$ is called the support of function $\mu$. To each term of a vague set, we have to attach a name or a label.

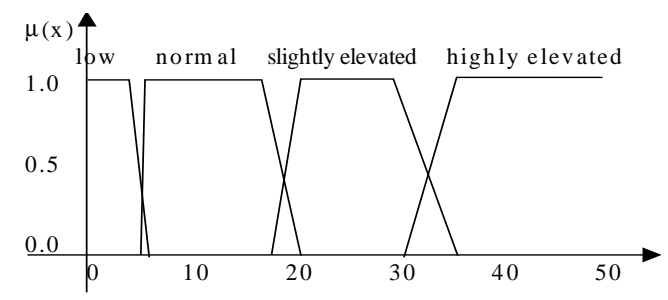

Fig. 4 The vague set of the linguistic variable SGOT

For the sake of an easy, robust interface for network initialization purposes (information stream from left to right in Fig. 3) it is wise not to assume any knowledge about appropriate membership functions by the user which is certainly true for most of the physicians. Instead, let us use the most simple membership function which is in coherence with the medical expert intuition: a simple trapezoidal bell shaped function which is directly assigned to the Radial Basis Function of a RBF neuron.

In conclusion, by using a trapezoidal function as standard membership function we can easily satisfy all the demands of the medical interface. As free parameters the lower and upper core limits [min,max] are chosen as the medical range limits while the ramps are assumed standard. An application of this kind of rule based system is presented in section 4.2. 


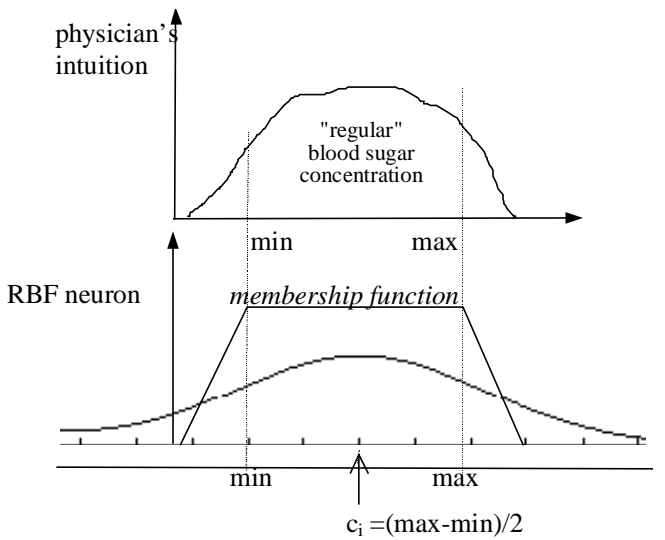

Fig. 5 Mapping the human association to RBF output

In [7] a prototype implementation Analystl [16] of such an interface is described.

\section{Case Study: Diagnosing Septic Shock Patients}

In intensive care units (ICUs) there is one event which only rarely occurs but which indicates a very critical condition of the patient: the septic shock. For patients being in this condition the survival rate dramatically drops down to $40-50 \%$ which is not acceptable.

Up to now, there is neither a successful clinical therapy to deal with this problem nor are there reliable early warning criteria to avoid such a situation. The event of sepsis and septic shock is rare and therefore statistically not well represented. Due to this fact, neither physicians can develop well grounded experience in this subject nor a statistical basis for this does exist. Therefore, the diagnosis of septic shock is still made too late, because at present there are no adequate tools to predict the progression of sepsis to septic shock. No diagnosis of septic shock can be made before organ dysfunction is manifest.

By the analysis of septic shock data we want to change this situation.

\section{The Data}

In our case, the epidemiology of 656 intensive care unit patients was elaborated in a study made between November 1995 and December 1997 at the clinic of the J.W.Goethe-University, Frankfurt am Main [28]. The data of this study and another study made in the same clinic between November 1993 and November 1995 is the basis of our work.

We set up a list of 140 variables, including readings (temperature, blood pressure, ...), drugs (dobutrex, dobutamin, ...) and therapy (diabetes, liver cirrhosis, ...). Our data base consists of 874 patients. 70 patients of all had a septic shock. 27 of the septic shock patients and 69 of all the patients deceased. 
With only a small amount of data in each study we had to fuse the two studies to one. Additionally, the variables had to be resampled in order to fit into a common time frame. For our data, not the typing errors but the missing values was the main problem.

The data base contains about 140 variables of metric nature, only partially usable. In our case, for analysis the physicians gave us recommendations which variables are the most important ones for a classification, based on their experience. The chosen variable set $V$ is composed of $n=16$ variables: $\mathrm{pO}_{2}$ (arterial) $[\mathrm{mmHg}], \mathrm{pCO}_{2}$ (arterial) $[\mathrm{mmHg}], \mathrm{pH}$, leukocytes [1000/ $\left.\mu \mathrm{l}\right]$, thromboplastin time (TPZ) $[\%]$, thrombocytes $[1000 / \mu \mathrm{l}]$, lactate $[\mathrm{mg} / \mathrm{dl}]$, creatinin $[\mathrm{mg} / \mathrm{dl}]$, heart frequency $[1 / \mathrm{min}]$, volume of urine $[\mathrm{ml} / 24 \mathrm{~h}]$, systolic blood pressure $[\mathrm{mmHg}]$, frequency of artificial respiratory [1/min], inspiratorical $\mathrm{O}_{2}$-concentration [\%], medication with antithrombine III AT3 [\%], medication with dopamine and dobutrex $[\mu \mathrm{g} /(\mathrm{kg} \cdot \min )]$.

\subsection{Diagnosis by growing neural networks}

The neural network chosen for our classification task is a modified version of the supervised growing neural gas (abbr. SGNG, see [10]). Compared to the classical multilayer perceptron trained with backpropagation (see [12]) which has reached a wide public, this network achieved similar results on classification tasks but converges faster, see [14]. The algorithm with our improvements and additional benchmark results are noted in detail in [11]. It is based on the idea of radial basis functions. Its additional advantage is the ability to insert neurons within the learning process to adapt its structure to the data.

In our case we had only 70 patients with the diagnosis "septic shock". Our classification is based on 2068 measurement vectors (16-dimensional samples) from variable set $V$ taken from the 70 septic shock patients. 348 samples were deleted because of too many missing values within the sample. With $75 \%$ of the 1720 remaining samples the SGNG was trained and with $25 \%$ samples from completely other patients than in the training set it was tested. The variables were normalized (mean 0 , standard deviation 1 ) for analysis.

The network chosen was the one with the lowest error on the smoothed test error function. Three repetitions of the complete learning process with different, randomly selected divisions of the data were made. The results are presented in Table 3.

Table 3 Correct classifications, sensitivity, specificity with standard deviation, minimum and maximum in $\%$ from three repetitions.

\begin{tabular}{|c|cccc|}
\hline measure & $\begin{array}{c}\text { mean } \\
\text { value }\end{array}$ & $\begin{array}{c}\text { standard } \\
\text { deviation }\end{array}$ & minimum & $\begin{array}{c}\text { maxi- } \\
\text { mum }\end{array}$ \\
\hline $\begin{array}{c}\text { correct } \\
\text { classification }\end{array}$ & 67.84 & 6.96 & 61.17 & 75.05 \\
sensitivity & 24.94 & 4.85 & 19.38 & 28.30 \\
specificity & 91.61 & 2.53 & 89.74 & 94.49 \\
\hline
\end{tabular}


To achieve a generally applicable result ten repetitions would be better, but here it is already clear: with the low number of data samples the results can only have prototypical character, even with more cleverly devised benchmark strategies.

On average we have an alarm rate (= 1 - specificity) of $8.39 \%$ for survived patients showing also a critical state and a detection of about 1 out of 4 critical illness states. For such a complex problem it is a not too bad, but clearly no excellent result. An explanation for this low number is grounded in the different, individual measurements of each patient.

\subsection{Diagnosis by rule based networks}

Results of classification procedures could provide a helpful tool for medical diagnosis. Nevertheless, in practice physicians are highly trained and skilled people who do not accept the diagnosis of an unknown machine (black box) in their routine. For real applications, the diagnosis machine should be become transparent, i.e. the diagnosis should explain the reasons for classification. Whereas the explanation component is obvious in classical symbolic expert system tools, neural network tools hardly explain their decisions. This is also true for the SGNG network used in the previous section.

Therefore, as important alternative in this section we consider a classification by learning classification rules which can be inspected by the physician. The details of the network structure and the learning algorithm can be found in [5],[19].

The result of the training procedure are rules of the form (belonging to the core or support rectangle)

$$
\begin{aligned}
& \text { if variable } 1 \text { in }(-\infty, 50) \text { and if variable } 2 \text { in }(20,40) \\
& \text { and if variable } 3 \text { in }(-\infty, \infty) \text { then class } l
\end{aligned}
$$

in addition with a classification. Here, variable 3 could be omitted.

Now we present the results of the rule generation process with our previously introduced septic shock data set. The data set is 16-dimensional. A maximum of 6 variables for every sample was allowed to be missing. The missing values were replaced by random data from normal distributions similar to the original distributions of the variables. So it was assured that the algorithm can not learn a biased result due to biased replacements, e.g. class-dependent means. We demand a minimum of 10 out of 17 variables measured for each sample, so there remained 1677 samples out of 2068 for analysis.

The data we used in 5 complete training sessions - each one with a different randomly chosen training data set - was in mean coming from class 1 with a percentage of $72.10 \%$ and from class 2 with a percentage of $27.91 \%$. In the mean 4.00 epochs were needed (with standard deviation 1.73, minimum 3 and maximum 7). Test data was taken from 35 randomly chosen patients for every training session, containing no data sample of the 35 patients in the training data set. In Table 4 the classification results are presented. 
Table 4 Mean, standard deviation, minimum and maximum of correct classifications and not classifiable data samples of the test data set. In \%.

\begin{tabular}{|l|c|c|c|c|}
\hline & mean & $\begin{array}{c}\text { standard } \\
\text { deviation }\end{array}$ & minimum & maximum \\
\hline correct classifications & 68.42 & 8.79 & 52.92 & 74.74 \\
\hline not classified & 0.10 & 0.22 & 0.00 & 0.48 \\
\hline
\end{tabular}

Average specificity ("deceased classified / all deceased") was $88 \%$ and average sensitivity ("survived classified / all survived") was $18.15 \%$. The classification result is not satisfying, although similar to the results in section 4.1 but with the benefit of explaining rules and less training epochs. Deceased patients were not detected very well. Reasons for this can be the very individual behavior of the patients and the data quality (irregularity of measurements, missing values). In this way it seems not possible to classify all the patients correctly, but it could be that in some areas of the data space the results are better (local rules). In the mean 22.80 rules were generated for the class survived and 17.80 rules were generated for class deceased.

\section{Discussion and Outlook}

After a short introduction and review of existing medical applications, the typical problems in analyzing medical data were presented and discussed.

In general, results of a patient classification or prediction task are true only with a certain probability. Therefore, any prognostic system can not predict always the correct future state but may just give early warnings for the treating physician. These warnings should constitute an additional source of information; the backward conclusion that, if there is no warning there is also no problems, is not true and should be avoided.

Two of the most typical and important neural network approaches were presented: the black-box and the neuro-fuzzy rule based system approach. The first approach for medical diagnosis by neural network is the black-box approach: A network is chosen and trained with examples of all classes. After successful training, the system is able to diagnose the unknown cases and to make predictions. The advantage of this approach is the adaptation of all parameters of the system for a (hopefully) best prediction performance.

Another diagnostic approach by neural networks is adaptive rule generation. By this, we can explain the class boundaries in the data and at the same time find out the necessary variables for a rule of the prediction system, see eq.(3). By using a special approach of rectangular basis networks we achieved approximately the same classification results as by the growing neural gas. Additionally, the diagnosis was explained by a set of explicitly stated medical rules.

One of the unresolved questions not only in this contribution is the application of the diagnostic results. Who should apply them when? Although a good test of the network provides the statistical means for the evaluation of the prediction performance, in clinical research this is not sufficient. A widely accepted proce- 
dure is a randomized double-blind study. Therefore, in order to make a prediction or classification system acceptable and usable in the medical world, after creating a successful neural network diagnosis system or deducing good rules a new clinical trial has to be conceived and performed with the final network state. Only after such an controlled study the results should be used, eventually taking the classification base and feasibility of such a system into account.

Even when the clinical trial was successful, you will encounter a problem: the problem of ignorance. As long as physicians can come along with their clinical routine in treating patients, they will do it. Nowadays, nearly for each disease there exist an international renowned standard procedure, but most physicians will not use it. Why? First, they have to know it and second, most physicians rely on their own expertise. To overcome this, one have to include diagnostic expertise into the clinical standard software. Here, clinical information systems with the possibility of plug-in software are very rare, but we are on the way. Future administrative necessities for complete input of patient data (e.g. TISS score) will also enhance the possibility for automatic diagnosis by such paradigms as neural networks.

\section{Acknowledgements}

The author would like to thank his coworkers of the MEDAN group F. Hamker for the results of section 4.1 and J. Paetz for the work of section 4.2.

\section{References}

[1] Baxt W. (1990): Use of an artificial neural network for data analysis in clinical decision making: the diagnosis of acute coronary occlusion, Neural Computation; 2: 480489

[2] Bottaci L., Drew P., Hartley J., Hadfield M., Farouk R., Lee P., Macintyre I., Duthie G., Monson J. (1997): Artificial neural networks applied to outcome prediction for colorectal cancer patients in separate institutions; Lancet, 350: 469-472

[3] Bounds D., Lloyd P.J.(1988): A multi-layer perceptron network for the diagnosis of low back pain; Proc. IEEE Int. Conf. on Neural Networks, Vol.II: 481-489

[4] Brause R. (1995): Neuronale Netze; Teubner Verlag, Stuttgart, 2nd edition

[5] Brause R., Hamker F., Paetz J.: Septic Shock Diagnosis By Neural Networks And Rule Based Systems; in: L.C. Jain: Computational Intelligence Techniques In Medical Diagnosis And Prognosis, Springer Verlag 2001, in press

[6] Brause R., Hanisch E. (Eds.) (2000), Medical Data Analysis ISMDA 2000. Springer Lecture Notes in Comp.Sc., LNCS 1933, Springer Verlag, Heidelberg

[7] Brause R., Friedrich F. (2000): A Neuro-Fuzzy Approach as Medical Interface, European Symp. On Neural Networks, ESANN 2000, pp. 201-206, D-Facto, Brussels

[8] de Dombal F.T., Leaper D.J., Staniland J.R., McCann A.P., Horrocks J.C. (1972): Acute Abdominal Pain, Brit. Med. J. 2: 9-13

[9] Forsstrom J.J., Dalton K.J. (1995): Artificial neural networks for decision support in clinical medicine. Ann Med. Oct; 27(5):509-17. 
[10] Fritzke B. (1995): A Growing Neural Gas Network Learns Topologies, Proc. Advances in Neural Information Processing Systems (NIPS 7), in G. Tesauro, D. S. Touretzky, T. K. Leen, MIT Press, Cambridge, MA, 625-632

[11] Hamker F., Paetz J., Thöne S., Brause R., Hanisch E. (2000): Erkennung kritischer Zustände von Patienten mit der Diagnose "Septischer Schock" mit einem RBF-Netz. Interner Bericht 04/00, Fachbereich Informatik, J.W. Goethe-Universität Frankfurt a. M., http://www.cs.uni-frankfurt.de/fbreports/fbreport04-00.pdf

[12] Haykin S. (1999): Neural Networks, A Comprehensive Foundation. Prentice Hall, 2nd edition, Upper Saddle River, NJ 07458

[13] Heden B. Edenbrandt L. Haisty W.K. jr., Pahlm O. (1994): Artificial neural networks for the electrocardiographic diagnosis of healed myocardial infarction; Am. J. Cardiol. 74(1): 5-8

[14] Heinke D., Hamker F. (1998), Comparing Neural Networks, A Benchmark on Growing Neural Gas, Growing Cell Structures, and Fuzzy ARTMAP. IEEE Transactions on Neural Networks 9(6), 1279-1291

[15] Hornik K., Stinchcombe M., White H. (1990): Universal Approximation of an Unknown Mapping and Its Derivatives Using Multilayer Feedforward Networks; Neural Networks, Vol 3, pp. 551-560,

[16] http://hiru.mcmaster.ca/

[17] http://www.cs.uni-frankfurt.de/ brause/software/Analyst1.exe

[18] http://www.ncbi.nlm.nih.gov:80/entrez/query.fcgi?db=PubMed

[19] Huber K.-P., Berthold M.R. (1995): Building Precise Classifiers with Automatic Rule Extraction. IEEE International Conference on Neural Networks 3, 1263-1268

[20] Zadeh L.A., Kacprzyk J. (1992): Fuzzy logic for the management of uncertainty, John Wiley \& Sons Inc., New York,

[21] McGonigal M. (1994): A New Technique for Survival Prediction in Trauma Care Using a Neural Network, Proc. World Conference on Neural Networks, pp.3495-3498

[22] Miller P.L. (1986): Expert Critiquing Systems: Practice-based Medical Consultation by Computer; Springer Verlag, New York

[23] Paetz J., Hamker F., and Thöne S. (2000): About the Analysis of Septic Shock Patient Data, in:[6]

[24] Penny W., Frost D. (1996): Neural networks in clinical medicine. Med. Decis. Making. Oct-Dec; 16(4):386-398.

[25] Sharpe P.K. Solberg H.E. Rootwelt K. Yearworth M. (1993): Artificial neural networks in diagnosis of thyroid function from in vitro laboratory tests; Clin. Chem. 39(11 Pt 1): 2248-2253

[26] Sharpe P.K., Caleb P. (1994): Artificial neural networks within medical decision support systems. Scand J Clin Lab Invest Suppl.;219:3-11.

[27] Snow P.B., Smith D.S. (1994): Artificial neural networks in the diagnosis and prognosis of prostate cancer: a pilot study; J. Urology. 152(5 Pt 2): 1923-1926

[28] Wade, S., Büssow, M, Hanisch, E. (1998): Epidemiology of Systemic Inflammatory Response Syndrome, Sepsis and Septic Shock in Surgical Intensive Care Patients, Chirurg;69(6):648-655 